\title{
DIFERENCIAS COGNITIVAS EN EL PROCESO DE APRENDIZAJE DE LA LECTURA
}

\author{
COGNITIVE DIFFERENCES IN THE PROCESS OF \\ LEARNING HOW TO READ \\ Francisco Sacristán Romero* \\ Universidad Complutense de Madrid
}

\section{RESUMEN:}

El objetivo principal se ha centrado en descubrir si los alumnos de enseñanza secundaria distinguen entre leer un texto y comprender su filosofía o ideas básicas. La metodología principal que se ha utilizado ha sido la realización de un cuestionario, basado en diferentes textos de prensa y su posterior codificación y análisis por grupos de edad. Respecto a los resultados y conclusiones más relevantes del estudio, describir que los lectores de menor edad y por ello, en principio, más novatos, realizan una lectura cuyos procesos son más simples, inmaduros y en algunos casos incompletos.

Palabras Clave: Lectura, Comprensión, Enseñanza Secundaria.

\begin{abstract}
:
The main objective has been centred in discovering if the students of secondary education distinguish between reading a text and understanding their basic philosophy or ideas. The main methodology has been the accomplishment of a questionnaire, based in different texts of press and their later codification and analysis by age groups. In relation to the results and conclusions more excellent of the study, to describe that the readers of smaller age and for that reason, in principle, more inexperienced, make a reading whose processes are simpler, immature and in some incomplete cases.
\end{abstract}

Key Words: Reading, Understanding, Secondary Education.

\footnotetext{
* Doctor en Ciencias de la Información y Licenciado en Ciencias de la Información, Psicología y Derecho. Profesor en la Facultad de Ciencias de la Información. Departamento de Historia de la Comunicación Social, líneas de investigación: Orientación psicoeducativa, Psicología de la lectura. E-mail: franciscosacristan@ozu.es
} 


\section{Introducción}

La decisión de elegir la realización de este trabajo para conocer la comprensión y asimilación de textos escritos por alumnos de Enseñanza Secundaria en el IES "Alameda de Osuna" del distrito de Barajas en Madrid no es algo gratuito ni que haya surgido por azar.

Se parte de la idea que leer como tal, supone asignar un significado a las palabras y, en esa dirección, comprender lo leído. Sin embargo, hay muchos y variados niveles, tanto cuantitativos como cualitativos, de comprensión y cabe plantearse si, en los de mayor complejidad léxica, sintáctica y semántica, es posible distinguir la capacidad de adquirir nuevos conocimientos mediante la lectura (leer para aprender) de la simple acción de leer (el que lee una novela o un periódico sin mayor interés que el propio reclamo del texto). Si se admite esta crucial distinción, más conceptual que real, sería posible enfrentarse a la identificación de las dificultades de ciertos alumnos.

Guiar a un alumno en su aprendizaje lector, es además de un objetivo relevante en sí mismo, un medio para alcanzar los demás objetivos educativos, donde las capacidades de autorregulación de los alumnos y la metacognición desempeñan un papel fundamental (Campanario y Otero, 2000, Barrero González, 2001). Las destrezas metacognitivas son especialmente importantes en el aprendizaje de las Ciencias, desde el momento que el alumno toma conocimiento de sus propias capacidades cognitivas y sobre la forma de regularlas (Campanario y Otero, 2000, Vargas y Arbeláez Gómez, 2001).

En virtud de ello y en consonancia con la opinión de numerosos investigadores del tema (Benito Morales 2000, Otero 1997, Sánchez Miguel 1993, Beach y Appleman 1984), se insiste en la importancia que tiene enseñarles a los alumnos estrategias para mejorar la comprensión lectora y prestar atención a cómo las emplean para organizar y controlar su propio aprendizaje.

Existen numerosos antecedentes en la investigación educativa que señalan la falta de comprensión lectora como una de las principales dificultades que tienen los alumnos para el logro de sus aprendizajes, especialmente por deficiencias en la forma de evaluar y controlar sus procesos cognitivos (Sánchez Miguel 1993, Otero 1997, Campanario y Otero 2000, Macías et al., 1999). La falta de comprensión se refleja en las limitaciones que ellos tienen para generalizar o transferir lo que han aprendido a situaciones diferentes a las que han originado su aprendizaje (Vargas y Arbeláez Gómez, 2001).

Los alumnos encuentran una especial dificultad cuando se enfrentan a la tarea de resumir y entresacar las ideas básicas de los textos literarios y periodísticos. Así, por ejemplo, parece que cuanto más clásicos son los autores leídos existen más obstáculos para comprender lo que pretenden decir al potencial lector. Un caso bastante peculiar y en el que es interesante detenerse es el del Quijote. El lenguaje de don Quijote es un buen compendio de su peculiar figura. Consecuente con su aspiración de ser tenido por un verdadero caballero adopta el estilo lingüístico del que se sirven los otros ejemplos literarios de Cervantes. Su castellano está impregnado de un léxico y una sintaxis típicamente medieval, que, lógicamente, extraña a más de un lector que ha hecho el intento de retomar la obra para acabarla de leer sin poder lograrlo.

El aspecto sui generis y particular de don Quijote es posible que, en principio, recaiga en la atmósfera descriptiva que recrea el lenguaje de la obra. El texto empieza presentando una idea de protagonista en la que don Quijote no se limita a su propio encumbramiento 
sino que depende de la acción, pues sólo a través de ella puede obtener la legitimidad y credibilidad como caballero andante. Escenas tales como aquellas en las que hace guardia en las ventas que él confunde con castillos, cuando ataca a la gente pacífica que le parece sospechosa, libera a prisioneros, etc., pueden parecer que son perfectamente comprensibles cuando se leen.

Quizás tanto en esta obra como en los editoriales de prensa que analizaron para este trabajo los alumnos de Secundaria el lector haya dejado olvidado un tanto el contexto de significación del texto. En don Quijote los actos que realiza rompen muchas de las expectativas de conducta que mantienen las personas que le rodean; se oponen, por lo tanto, al comportamiento esperable en el espacio público del libro y a las convenciones cotidianas que aseguran la estabilidad social. El idioma ceremonial de don Quijote - su lanza, el escudo, su armadura, su habla y sus maneras-, en síntesis, todos los símbolos que él muestra con orgullo, le estigmatizan como una persona desviada. El caballero dispone de un repertorio de signos, reglas y modos de comportamiento que no coinciden con los de su entorno.

Su locura no reside en el contenido implícito de las actitudes que defiende, sino en la perseverancia que muestra cuando quiere imponerlas en una era post-caballeresca. Con rigor, su propuesta de reclutar una tropa de caballeros españoles para luchar contra los turcos no puede ser considerada como un delirio. Ciertamente, don Quijote se mueve en un mundo construido de elementos ficticios, que en tal forma probablemente nunca existió en la realidad, pero las hazañas grandiosas de los caballeros que él rememora, se desarrollan en un escenario medieval, un marco de referencia que don Quijote integra en su sistema mental. Se comporta como si la Edad Media, con su orden feudal, sus normas y valores, continuase existiendo y vuelve, de este modo, la espalda a las transformaciones del siglo XVI.

¿Se puede contemplar alguna semejanza cuando se leen editoriales sobre un asunto alejado del entorno cotidiano y especialmente mental, como es el conflicto de Kosovo, con lo expuesto hasta aquí acerca de don Quijote?

Quizás una de las peculiaridades de ambas situaciones es que los lectores tienen importantes problemas de comprensión por ser desconocedores de los contextos en que se ubican las circunstancias de los asuntos que leen.

El destino de toda comunicación es modificar, ampliar o confirmar la visión del mundo. Los textos y los discursos constituyen una invitación para construir mundos y sus destinatarios así debieran entenderlo, confrontando lo que ya se sabe de esa cercana o lejana realidad con la información que están en ellos. Pero este diálogo o interacción sólo puede tener lugar en la medida en que sea posible introducirse en el mensaje y, por tanto, siempre que se reconozca y recorra su estructuración interna. Todo ello, resulta especialmente relevante en el caso del texto escrito, dado que la comunicación a través del soporte de la escritura tiene en relación a la oral un mayor potencial grado de integración y descontextualización (a pesar de que tanto los pasajes de don Quijote o los editoriales sobre conflictos parezcan tan unidos a un espacio y tiempo concretos).

Por todo ello, ¿qué significa considerar atentamente lo que está en el texto? Dicho de otro modo: ¿cómo es posible percibir el verdadero sentido de un texto?

Independientemente que se trate de un texto largo o pequeño, los escritos precisan una continuidad en los asuntos que abordan. En la conversación cotidiana, los hablantes poseen múltiples recursos para indicar a la audiencia los cambios temáticos. 
Ante los textos, el lector debe hacer lo propio, pero con el problema añadido de enfrentarse a evidencias menos directas e impersonales. Es también constatable que, tanto en los textos como en los discursos ordinarios, la información no siempre ocupa o posee la misma relevancia o significatividad.

\section{Método}

\section{Objetivos}

Los objetivos de este trabajo con los estudiantes de Enseñanza Secundaria del IES "Alameda de Osuna" son los siguientes, a saber:

1. Verificar si los alumnos de mayor edad extraían información más o menos sustantiva y no arbitraria respecto a sus compañeros de curso inferiores, basando la premisa en "la aparente mayor experiencia lectora" de los primeros.

2. Analizar la interpretación que los alumnos daban a los editoriales de acuerdo al condicionamiento de los conocimientos previos del lector sobre la guerra de Kosovo y los procesos cognitivos desarrollados.

3. Intentar averiguar cómo comprenden los alumnos los textos presentados:

a) A través de una "comprensión literal", referida a la recuperación de la información explícitamente descrita en el texto, sin añadir ni omitir datos.

b) A través de una "comprensión inferencial", consistente en extrapolar información no expresada explícitamente en el texto.

4. Esperar la posibilidad de la emisión de juicios críticos valorativos y comparaciones entre lo que los editoriales expresan y otros criterios externos o internos de los lectores.

La guerra de Kosovo es un asunto suficientemente complejo y abierto en cuanto a su solución que puede desencadenar relevantes comentarios críticos por parte de los estudiantes de Secundaria.

5. Analizar las estrategias puestas en marcha por los alumnos para titular y hacer los resúmenes correspondientes de cada uno de los editoriales.

6. Indagar en las dificultades o facilidades encontradas en las respuestas de los alumnos respecto a la coherencia temática del texto y su recuerdo en función del número de cadenas causales entre párrafos de un mismo editorial que puedan reproducir.

Tomando en consideración la relevancia que las cadenas causales tienen para este objetivo, se puede indicar que si la estructura va presentándose ordenadamente a lo largo del texto se reducirá la dificultad de comprensión del texto.

7. Comprobar si concebían, de acuerdo a sus respuestas, la comprensión de la lectura como una compleja actividad cognitiva del procesamiento de la información, o por el contrario, como una mera acumulación de datos más "sintáctica" que semántica.

\section{Diseño}

Se ha elegido el análisis de casos por el planteamiento de unas hipótesis en las que resulta mucho más apropiado este diseño que cualquier otro porque permite caracterizar 
adecuadamente el problema de acuerdo a las variables de tiempo, lugar y personas de la muestra.

\section{Sujetos}

El interés del trabajo está centrado en conocer el grado y nivel de comprensión de lectura de los editoriales de prensa escrita acerca del conflicto de Kosovo por alumnos de Enseñanza Secundaria.

Los adolescentes que respondieron a los cuestionarios tenían edades comprendidas entre 12 y 20 años, aunque al final se decidió solamente incluir a aquellas alumnas y alumnos con edades entre 14 y 20 años inclusive, dado que en las chicas y chicos de 12 y 13 años se constataron serias dificultades para responder a alguna de las preguntas planteadas.

De los 40 cuestionarios inicialmente entregados para realizar esta tarea, se consideraron como válidos 28, descartando los 12 restantes debido a las razones anteriormente expuestas acerca de los alumnos de 12 y 13 años. Por lo tanto, la base de la muestra se ha configurado en torno a 28 alumnas y alumnos entre 14 y 20 años, de $3^{\circ}$ de ESO y COU mayoritariamente, del Instituto de Enseñanza Secundaria "Alameda de Osuna" de Madrid, de los que 20 eran mujeres y 8 eran varones, con la siguiente distribución por edades:

- 14 años: 8 alumnos.

- 15 años: 8 alumnos.

- 16 años: 1 alumno.

- 18 años: 3 alumnos.

- 19 años: 6 alumnos.

- 20 años: 2 alumnos.

La selección específica de la muestra se llevó a cabo por los mismos profesores del IES "Alameda de Osuna" a los que se les solicitó encarecidamente que consiguieran distribuir los cuestionarios de forma proporcional entre las distintas edades y cursos. Teniendo en cuenta algunos de los obstáculos que tuvieron con chicas y chicos de las edades inferiores de la escala, se decidió que los cuestionarios de estas personas no fueran incluidos para realizar el análisis posterior porque se podían introducir variables extrañas no controladas en los datos y con ello posiblemente se incurriera en algunos sesgos difíciles de superar conforme se avanzase en el tratamiento de toda la información aportada.

\section{Instrumentos}

Se elaboró un material escrito que incluía un apartado de lectura de los tres editoriales de los diarios LA RAZÓN, EL MUNDO y EL PAÍS del 5 de Abril de 1999, seleccionados para la tarea y cuatro restantes que se referían a la contestación de una serie de preguntas abiertas que iban de menor a mayor complejidad según se avanzaba. Estas preguntas se elaboraron con la pretensión de analizar las estrategias para la extracción de ideas principales, agilidad y claridad a la hora de poner títulos breves a cada uno de los párrafos de cada editorial, comentarios específicos acerca de lo expuesto por los editorialistas en cada uno de los párrafos y finalmente ver cómo procesaban los alumnos la información cuando se les pedía un resumen final de cada editorial. 
Las instrucciones de la tarea seguían el siguiente orden:

1. Lectura de los tres editoriales subrayados referentes al tema: Guerra de Kosovo.

Se pedía a los alumnos que hiciesen una primera lectura de los tres textos, cada uno con su ritmo particular e intentando concentrar al máximo su atención en la tarea. Los profesores explicaron a los alumnos que el objetivo de este primer contacto con los editoriales era hacerse una idea general de cada uno de ellos.

Les dijeron que si alguna palabra les resultaba desconocida en cuanto a su significado debían preguntar para aclarar esas dudas desde un principio, o en su defecto, consultar los términos dudosos en un diccionario.

2. Extraer de cada párrafo la idea que se considere principal. ¿De qué trata el párrafo?

En este paso los profesores explicaban de antemano a los alumnos que lo importante era que hiciesen una segunda lectura del texto con detenimiento en cada uno de los párrafos para ver cuál era, desde la perspectiva de cada uno de los alumnos, el asunto central sobre el que guían las ideas contenidas en cada párrafo.

3. Poner un título a cada párrafo.

Para ayudar a las chicas y chicos a una comprensión mejor de la anterior tarea, podía ser útil el que titularan cada uno de los párrafos. Se insistió a los profesores que en esta fase era muy importante hacerles ver a los alumnos que un "título no es equivalente a un resumen" y que les recordasen que el título tan sólo requiere en muchas ocasiones una palabra o un par de palabras.

Lo realmente esencial era que los alumnos entendiesen que esas palabras empleadas en los titulares debían ser un reflejo del contenido de cada párrafo.

Una vez aclarado el sentido de los pasos 2 y 3 , los profesores recalcaron a los alumnos que cuando tuviesen un esquema mental definido sobre qué escribir en estas preguntas acerca de la idea de cada párrafo y el título particular que ellos pondrían, redactasen todo en hojas aparte del material entregado para facilitar la labor de análisis de todas sus respuestas.

Por supuesto, se contaba con que algunos alumnos en vez de un título iban a escribir un resumen. Un ejemplo bien elocuente se encuentra en los títulos que entregó José Luis, alumno de COU y de 18 años:

- Título del editorial de LA RAZÓN: "Fuerzas terrestres, para los albanokosovares, de los aliados".

- Título del editorial de EL MUNDO: "Graves consecuencias tras un error de la OTAN".

- Título del editorial EL PAÍS: "Organización de la ayuda para evitar la catástrofe".

Por otra parte, se comprobó que algunos de los alumnos se ahorraban el trabajo de escribir lo que se les pedía en la pregunta 2 de las instrucciones de la tarea y empleaban la técnica del "subrayado" para extraer de cada párrafo la idea que consideraban principal. Este método tenía el inconveniente que contestaban a la pregunta con las mismas palabras que habían usado los editorialistas; de esta forma, no existía la explicación de su elección a través de las propias palabras de cada uno de los alumnos que optaron por el subrayado. 
Otro error percibido en varias personas consistía en que elegían como título el primer término que aparecían en cada uno de los párrafos. Este fallo fue cometido a pesar de que los profesores previamente a la realización de la tarea les matizaron que un buen título es algo cuyo contenido debe estar presente en todas las oraciones del párrafo, y no sólo al principio, en el medio o al final de párrafo.

4. Desglosar los comentarios de cada párrafo: ¿Qué se dice del tema?

5. Resumen de cada editorial:

5.1. ¿Se puede quitar algo porque ya se sabe muy bien o porque se dice de varias maneras?

5.2. ¿Hay algo en el texto que se pueda considerar como un ejemplo de algo que ya se conoce?

5.3. ¿Es posible sustituir todo esto por alguna palabra que "diga lo mismo"?

\section{Procedimiento}

El material de trabajo era anónimo y fue repartido por distintos profesores del IES “Alameda de Osuna" a sus alumnas y alumnos. A pesar de insistir en el anonimato, hubo un significativo número de estudiantes que pusieron su nombre y dos apellidos en las hojas entregadas.

La contestación a las preguntas planteadas se realizó en las clases correspondientes a la asignatura de Lengua durante el mes de Abril de 1999, coincidiendo con la parte del programa referente a "Lenguaje periodístico" que los profesores explicaron a estos alumnos antes de pasarles los materiales.

Esto les sirvió a ellos mismos para conocer de primera mano el grado de comprensión de alguna de las lecciones de "Lenguaje periodístico" en la modalidad más práctica que planteaban las preguntas del cuestionario.

Siendo plenamente conscientes de los objetivos del estudio y las características de una gran parte de las variables incluidas, se decidió realizar un análisis de correspondencias múltiples entre las contestaciones a las diversas preguntas.

El procedimiento buscaba reducir la variabilidad expuesta en los contenidos originales del trabajo a un número menor de dimensiones con el propósito de investigar las semejanzas y diferencias de toda la información posible que escribieron los alumnos.

Este modus operandi podía ser útil para construir una agrupación o clasificación más realista de los sujetos de la muestra en función de sus peculiaridades particulares, ofreciendo información adicional sobre las características que guían sus maneras de procesar los datos de los editoriales respecto a la comprensión de las ideas principales y secundarias de los textos y expresar resúmenes precisos y sin vaguedades de cada uno de los editoriales.

\section{Conclusiones}

Tras el análisis de los resultados, se puede sostener que las hipótesis de trabajo se han confirmado en su mayoría. 
Así, los lectores de menor edad y por ello, en principio, más novatos, realizan una lectura cuyos procesos son más simples, inmaduros y en algunos casos incompletos mientras que los lectores de mayor edad y por ende, teóricamente más expertos, realizan una lectura que implica procesos cognitivos más complejos, adecuados y profundos.

De acuerdo a los objetivos de partida, lo anteriormente expuesto se podría concretar en los siguientes puntos:

- Los lectores jóvenes suelen realizar resúmenes literales utilizando en muchos casos la técnica del subrayado. Los de más edad, por el contrario, hacen resúmenes en los que traducen el contenido del editorial a sus propias palabras, dándole una mayor amplitud a la información manejada.

- Del punto anterior se desprende el hecho que los primeros expresan ideas incorrectas. Esto, sin embargo, no es frecuente verlo en el segundo grupo.

- Los más novatos parecen, en muchas ocasiones, que no son capaces de reconocer la conexión existente entre los diferentes párrafos sin poder, de esta forma, establecer relaciones causa-efecto, hecho que si se percibe en los más expertos.

- Por último, los novatos no intentan integrar el conocimiento que tienen de otras áreas, a diferencia de los expertos. Esto constituye otra razón por la que los novatos no llegan a la comprensión de la lectura.

No obstante, es necesario puntualizar que estos resultados no se dan en todos los casos; como siempre, es posible encontrar diferencias individuales que podrían ejemplificarse en las personas de Pablo y Juan Jesús, de 20 años. Estos chicos se acercan más que a un nivel avanzado, a uno intermedio de comprensión. No logran entresacar las ideas centrales de los textos, realizando inferencias incorrectas y estando bastante apegados a la literalidad de los contenidos.

Pero también, es posible encontrar diferencias individuales en los grupos de "novatos", como puede ser el caso de Luna de 15 años cuyas ideas centrales están bien conseguidas, basando sus decisiones en procesos más maduros.

Respecto a las inferencias realizadas, también se encuentran importantes diferencias particulares entre las alumnas y alumnos. Sorprende encontrar que sean los más jóvenes de la muestra los que presentan posiciones más valorativas en contraposición con los mayores de la muestra que parecen han sido más objetivos y cautelosos en sus opiniones, sin hacer juicios de valor por doquier.

No obstante, si se analiza con más profundidad los juicios que realizan, esta primera sorpresa se torna en algo que según lo expuesto en el cuerpo teórico se podía predecir. Es referente a que los juicios que realizan se desprenden de sus ideas implícitas y de la información literal del texto. En definitiva, no han integrado y construido su propia versión de la realidad en función de lo expuesto en los editoriales y sus ideas implícitas. Dicho de otra forma: se han dejado llevar por uno de los dos factores, sin ejercitar una visión crítica e integradora de los diversos conocimientos como parece recomendable en este caso. Algunos ejemplos ilustrativos son cuando una de las chicas de la muestra manifiesta que "La guerra es una basura" o cuando otra habla sobre Clinton, etc.

Otro aspecto relevante es el hecho que los sujetos de 11 y 12 años tuvieron que ser descartados. Esto también se puede interpretar como un dato a favor de las hipótesis del trabajo, comprobando que los más jovencitos e inexpertos de la muestra inicial no tienen a 
su disposición o no logran poner en marcha los mecanismos requeridos para este tipo de lectura.

Para terminar, una reflexión sobre el hecho que son pocos los sujetos que han intentado resumir el tercer editorial y muchos de los que lo hacían era a través de la técnica del subrayado, a pesar que no hubieran utilizado ésta con los otros dos editoriales. Una posible causa de esto pudo ser el no dar con la selección del editorial adecuado conforme a los cuatro criterios utilizados. Ningún editorial cumplía totalmente las exigencias que requerían los cuatro criterios mencionados; por eso, se decidió centrar la elección en las descripciones-diagnóstico de la situación y en las interrelaciones entre los distintos argumentos empleados.

\section{Referencias Bibliográficas}

Barrero González N. (2001). "El enfoque metacognitivo en la Educación”. Revista Electrónica de Investigación y Evaluación Educativa, 7 (2). Consultado el 8 de agosto de 2002 en: http://www.uv. es/RELIEVE/v7n2/RELIEVE/v7n2_0.htm

Beach, R. and Appleman, D. (1984). "Reading strategies for expository and literary text types". En A. C. Purves and O. S. Niles (eds). Becoming readers in a complexity society. University of Chicago Press.

Benito Morales, F. (2000). "La alfabetización en información en centros de primaria y secundaria". En: Estrategias y Modelos para enseñar a usar la información. Murcia KR. Consultado el 24 de noviembre de 2001 en: http://www.gtil.edu.um.es:8080/jgomez/nei/intranet/comprensión

Bernárdez, E. (1982). Introducción a la lingüistica del texto. Madrid: Espasa Calpe.

Campanario J. M. y Otero J. C. (2000). "Más allá de las ideas previas como dificultades de aprendizaje: Las pautas de pensamiento, las concepciones epistemológicas y las estrategias metacognitivas de los alumnos de ciencias". Enseñanza de las Ciencias, 18 (2): 161-169.

Coll, C. (1989). Conocimiento psicológico y práctica educativa. Barcelona: Barcanova.

Edwards, D. y Mercer, N. (1987). El desarrollo de la comprensión en el aula. Barcelona: Paidos/MEC.

García Madruga, J. C. y Martín Cordero, J. I. (1987). Aprendizaje.Comprensión y Retención de Textos. Madrid: Estudios de Educación a Distancia.

Gelb, I. J. (1976). Historia de la escritura. Madrid: Alianza.

López Rodriguez, N. (1982). Cómo valorar textos escolares. Madrid: Cincel.

Luria, A. R. (1980). Conciencia y Lenguaje. Madrid: Pablo del Río Editor.

Macías A., Castro J. y Maturano, C. (1999). "Estudio de algunas variables que afectan la comprensión de textos de física”. Enseñanza de las Ciencias, 17 (3): 431- 440.

Mayor, J. (1984). "Texto y discurso", en J. Mayor (Ed.). Psicología del pensamiento y del lenguaje. Vol. I. Madrid: UNED.

Otero, J. (1990). "Variables cognitivas y metacognitivas en la comprensión de textos científicos: el papel de los esquemas y el control de la propia comprensión". Enseñanza de las Ciencias, 8: $17-22$.

Otero, J. (1997). "El conocimiento de la falta de conocimiento de un texto científico. Alambique". Didáctica de las Ciencias Experimentales, 11: 15-22.

Puente, A. (1991). Comprensión de la lectura y acción docente. Madrid: Pirámide. 
Sánchez Miguel, E. (1993). Los textos expositivos. Estrategias para mejorar su comprensión. Editorial Santillana. España.

Vargas E. y Arbeláez Gómez C. (2001). "Consideraciones teóricas acerca de la metacognición”. Revista de Ciencias Humanas, 28. Consultado 19 de junio de 2002 en: http://www.utp.edu.co/ chumanas/revistas/revistas/rev28/vargas.htlm

Fecha de recepción: 06/01/06

Fecha de revisión: 15/09/07

Fecha de aceptación: 27/02/08 